\title{
Evidence for enhanced tissue factor expression in age-related macular degeneration
}

\author{
Youngeun Cho ${ }^{1}$, Xiaoguang Cao ${ }^{1,2}$, DeFen Shen ${ }^{1}$, Jingsheng Tuo ${ }^{1}$, Leonard M Parver ${ }^{3}$, Frederick R Rickles ${ }^{4}$ \\ and Chi-Chao Chan ${ }^{1}$
}

Tissue factor (TF) is the primary initiator of blood coagulation. In addition to hemostasis, TF can initiate intracellular signaling and promote inflammation and angiogenesis, the key processes underlying the pathogenesis of age-related macular degeneration (AMD). AMD, the leading cause of irreversible blindness among the elderly, involves many genetic and environmental risk factors, including oxidative stress and inflammation. In this study, TF expression was examined in human AMD tissue and in the eyes of a model of AMD, the $C \mathrm{Cl}^{-/-} / \mathrm{C} \times 3 \mathrm{Cr}^{-/-}$(DKO) mouse, as well as in the ARPE-19 cell line after lipopolysaccharide (LPS) and $\mathrm{H}_{2} \mathrm{O}_{2}$ stimulation. Total RNA was extracted from tissue samples and further analyzed by real-time RT-PCR. Immunohistochemistry was performed to evaluate TF protein expression. In the human retina, a 32-fold increase of TF mRNA expression was detected in AMD macular lesions compared with normal maculae. TF protein expression was also enhanced in human AMD maculae. Similarly, TF transcript and protein expression were moderately increased in retinal lesions, neuroretinal tissue, and cultured RPE cells of DKO mice compared with agematched wild-type mice. TF expression level correlated with age in both wild-type and DKO mice. In order to better understand how AMD might lead to enhanced TF expression, 1, 5, and $10 \mu \mathrm{g} / \mathrm{ml} \mathrm{LPS}$ as well as 100 and $200 \mu \mathrm{M} \mathrm{H}_{2} \mathrm{O}_{2}$ were used to stimulate ARPE-19 cells for 24 and $2 \mathrm{~h}$, respectively. LPS treatment consistently increased TF transcript and protein expression. $\mathrm{H}_{2} \mathrm{O}_{2}$ alone or in combination with LPS also moderately enhanced TF expression. These results indicate that upregulated TF expression may be associated with AMD, and inflammatory and oxidative stress may contribute to TF expression in AMD eyes.

Laboratory Investigation (2011) 91, 519-526; doi:10.1038/labinvest.2010.184; published online 1 November 2010

KEYWORDS: age-related macular degeneration; inflammation; oxidative stress; retina; retinal pigment epithelium; tissue factor

Age-related macular degeneration (AMD) represents a leading cause of irreversible blindness in the elderly. ${ }^{1,2}$ Advanced AMD is generally subclassified into geographic atrophy (dry) and neovascular/exudative (wet) AMD. Dry AMD progresses relatively slowly and is characterized by the accumulation of drusen deposits, degeneration, and atrophy of both retinal pigment epithelium (RPE) and photoreceptors. Wet AMD leads to sudden and severe vision loss and is characterized by choroidal neovascularization $(\mathrm{CNV})$ - the growth of new blood vessels from the choroid into Bruch's membrane or the subretinal space and retina.

Tissue factor (TF), a transmembrane cell-surface receptor for plasma coagulation factor VII (FVII) and its activated form FVIIa, is the primary initiator of mammalian blood coagulation. Normal endothelium lacks detectable TF expression, whereas vascular subendothelial cells constitutively express TF. ${ }^{3}$ Upon vascular injury, FVII binds to TF to form the TF/VIIa complex, which initiates the coagulation cascade by activating downstream coagulation factors. In addition to hemostasis, the TF/VIIa complex can also mediate intracellular signaling through protease-activated receptors, and promote inflammation ${ }^{4,5}$ and angiogenesis. ${ }^{6-8}$

Inflammation and angiogenesis have been implicated in the pathogenesis of AMD. Drusen formation initiates inflammation by stimulating the production of cytokines and reactive oxygen species (ROS), and leads to further RPE and

\footnotetext{
${ }^{1}$ Immunopathology Section, Laboratory of Immunology, National Eye Institute, National Institutes of Health, Bethesda, MD, USA; ${ }^{2}$ Department of Ophthalmology, People's Hospital, Peking University, Beijing, China; ${ }^{3}$ Department of Ophthalmology, Georgetown University Medical School, Washington, DC, USA and ${ }^{4}$ Departments of Medicine, Pediatrics, Pharmacology and Physiology, The George Washington University School of Medicine and Health Sciences, Washington, DC, USA Correspondence: Dr C-C Chan, MD, Immunopathology Section, National Eye Institute, National Institutes of Health, 10 Center Drive, 10/10N103, Bethesda, MD 20892-1857, USA.
}

E-mail: chanc@nei.nih.gov

Received 12 June 2010; revised 31 August 2010; accepted 24 September 2010 
photoreceptor damage. Studies have found an association of AMD with single-nucleotide polymorphisms of the genes coding for complement factor $\mathrm{H}$, factor $\mathrm{B}, \mathrm{C} 2$, and C3. ${ }^{1,9}$ Of interest, coagulation factor $\mathrm{X}$ and fibrinogen, which are downstream mediators of blood coagulation closely related to the expression of TF activity, have also been detected in AMD lesions. ${ }^{10-14}$ Inflammation elicited by lipopolysaccharide (LPS), TNF- $\alpha$, IL-1, IL-6, C5a, and many other factors can result in increased cellular expression of TF. Enhanced TF expression may in turn induce expression of proinflammatory cytokines such as IL- $1 \beta$, IL-6, IL-8, and macrophage inflammatory protein- $2 \alpha$ (MIP- $2 \alpha /$ CXCL $2 \alpha){ }^{4,15}$

Analogous to the process of tumor angiogenesis, in the later stages of wet $\mathrm{AMD}, \mathrm{CNV}$ serves to actually reduce the blood supply to the retina, with diminished transport of macromolecules such as oxygen, thereby creating a hypoxic environment. Hypoxia subsequently induces the expression of vascular endothelial growth factor (VEGF) and promotes further $\mathrm{CNV}$ formation. ${ }^{16}$ In cancer and inflammation, a positive feedback loop exists between VEGF and TF; TF can induce angiogenesis by upregulating VEGF, ${ }^{17-19}$ and enhanced VEGF can in turn increase TF expression. ${ }^{20}$

Recent studies have suggested the possibility that TF may be implicated in the pathogenesis of AMD. ${ }^{14}$ Immunostaining revealed TF expression in RPE cells and macrophages in post-mortem eyes with CNV, and in surgically excised CNV specimens, ${ }^{21} \mathrm{TF}$ was expressed strongly in macrophages and variably in RPE cells. In addition, TF staining was observed to be increased in 'inflammatory-active' versus 'inflammatory-inactive' CNV. Belting et al reported that TF phosphorylation was associated with pathological neovascular vessels but not with normal vessels in human diabetic retina, indicating a potential role of TF in CNV formation. Furthermore, targeting TF has been proposed as a potential immunotherapy for treating CNV. Administration of a FVII-Fc chimeric antibody, which targets TF, selectively obliterated $\mathrm{CNV}$ without any side effects in laser-induced mouse and pig models, which simulate neovascular $\mathrm{AMD}^{22,23}$ Taken together, these findings suggest that aberrant expression or activity of intraocular TF may have a role in AMD pathogenesis, and that TF may serve as an effective therapeutic target for pathological neovascular lesions in AMD retina.

$\mathrm{TF}$ expression in normal and diseased eyes as well as its association with increasing age has not been previously demonstrated. In addition, it still remains unclear whether $\mathrm{TF}$ can serve as a target or marker in early and dry AMD. In this study, we examined TF expression in human AMD retina, as well as in the eyes of the murine model of AMD, $\mathrm{Ccl}^{-/-} / \mathrm{C} \times 3 \mathrm{crl}^{-1-}$ double-knockout (DKO) mice, which spontaneously develop focal, progressive retinal lesions, and elevated A2E levels as early as at 4-6 weeks of age. ${ }^{24} \mathrm{TF}$ expression under the control of inflammatory and oxidative stress was also evaluated in vitro in ARPE-19 cells after stimulation with LPS and $\mathrm{H}_{2} \mathrm{O}_{2}$.

\section{MATERIALS AND METHODS}

\section{Human Eye Sections}

The study was approved by the National Eye Institute Institutional Review Board for human subjects. Archived paraffin-embedded sections cut through the macula of agematched two non-AMD and four AMD eyes were selected. The AMD retinas demonstrated macular disciform scars or neovascular AMD, indicating end stage of the disease.

\section{Animals}

The development of DKO mice was described previously. ${ }^{24,25}$ The DKO mice and age-matched wild-type (WT) control (C57Bl/6) mice were bred in-house. All animal experiments were performed under protocols approved by the NEI institutional animal care and use committee and were in compliance with the ARVO Statement for the use of animals in ophthalmic and vision research. Both WT and DKO had young (1-month-old mice) and old (1-year-old mice) subgroups. Three mice of each subgroup were used in experiments.

\section{Cell Culture}

Adult human RPE cells (ARPE-19) were obtained from the American Type Culture Collection (Manassas, VA, USA) and cultured in DMEM/F12 medium (1:1) (Sigma, St Louis, MO) containing $10 \%$ fetal bovine serum (FBS; Sigma-Aldrich, St Louis, MO), 1\% L-glutamine-penicillin-streptomycin (Sigma-Aldrich), 1\% $100 \times$ MEM nonessential amino acids (Invitrogen, Carlsbad, CA, USA), and 1\% N1 growth medium supplement (Sigma-Aldrich). The cells were cultured at $37^{\circ} \mathrm{C}$ in humidified $5 \% \mathrm{CO}_{2}$ condition and split when $90 \%$ confluent.

\section{Microdissection}

Human retinal cells in the macular area, including RPE, were manually microdissected from paraffin-embedded AMD eye sections. Retinal neuronal and RPE cells from normal maculae were also microdissected from non-AMD human eye sections to compare TF mRNA expression levels. Retinal lesion sites of DKO mice and comparable retinal areas from WT mice were microdissected from frozen sections. Total RNA was isolated from the microdissected cells following the instructions of the Picopure ${ }^{\mathrm{TM}}$ RNA Isolation Kit (Arcturus Bioscience, Mountain View, CA, USA).

\section{Dissection of Mouse Neuroretinal Tissue and RPE}

The neuroretinal tissue and RPE of DKO and WT mice was dissected following a protocol published previously. ${ }^{26}$ In brief, a limbal puncture was made in an enucleated eye. Cutting around the limbus, the cornea, lens, and vitreous were removed. Growth medium was injected to dislodge the neuroretina from the RPE. The neuroretina was plucked off and snap-frozen for RNA isolation. A total of $100 \mu \mathrm{l}$ of $0.25 \%$ EDTA-trypsin (Invitrogen) was added to each posterior eyecup and incubated at $37^{\circ} \mathrm{C}$ for $45 \mathrm{~min}$. The RPE cells were 
dislodged by injecting growth media. The collected RPE was spun at 2000 r.p.m. for $5 \mathrm{~min}$. The cell pellet was resuspended in $5 \mathrm{ml}$ growth medium and plated onto a 96-well cell culture plate (Costar, Corning, Corning, NY, USA) at $5 \% \mathrm{CO}_{2} / 37^{\circ} \mathrm{C}$. The first change of growth medium was performed after $72 \mathrm{~h}$. Thereafter, growth medium was changed every other day and cells split in a 1:3 ratio into new $25 \mathrm{~cm}^{2}$ flasks upon reaching $90 \%$ confluence. The cells were used for experiments at passages between 4 and 7 .

\section{Detection of TF Transcripts by Quantitative Real-Time RT-PCR}

Total RNA was isolated from microdissected retinal tissue, cultured mouse RPE cells, and ARPE-19 cells using the PureLink Micro-to-Midi RNA purification system according to the manufacturer's protocol (Invitrogen). Equal amounts of RNA were reverse transcribed with Superscript II RNase H Reverse Transcriptase (Invitrogen). RT-PCR was performed on the resulting cDNA using Brilliant SYBR Green QPCR Master Mix (Stratagene, La Jolla, CA, USA). The comparative cycle threshold value $(\mathrm{Ct})$ method, representing log transformation, was used to establish relative quantification of the fold changes in gene expression using Stratagene $\mathrm{M} \times 3000 \mathrm{P}$ QPCR System (SABiosciences, Frederick, MD, USA). Fold changes were normalized first by the level of $\beta$-actin. The average fold change in different subgroups was again normalized to the level of young WT or control cells. TF primers were purchased from SABiosciences. For an internal control, $\beta$-actin was amplified with a sense primer ( $5^{\prime}$-TCCCCCAAC TTGAGATGTATGAAG- $3^{\prime}$ ) and an antisense primer (5'AACTGGTCTCAAGTCAGTGTACAGG-3') (Sigma-Genosys, Woodlands, TX, USA).

\section{Immunohistochemistry}

Immunohistochemical (IHC) analysis was carried out using avidin-biotin complex immunoperoxidase technique. Paraffin-embedded human eye sections were deparaffinized in xylene and rehydrated in a graded series of ethanol before IHC. Enucleated WT and DKO mouse eyes were embedded in optimal cutting temperature (OCT) compound (Sakura Finetek, Torrance, CA, USA), snap-frozen, and cut via the papillary-optic nerve plane. Cultured mouse RPE cells and ARPE-19 cells were plated overnight on eight-well glass LabTek chamber slides (Nunc, Rochester, NY, USA) at 10000 cells/well.

The specimens were fixed in acetone for $7 \mathrm{~min}$ and rinsed in Tris-buffer saline (0.05 M, pH 7.4). Endogenous peroxide activity was blocked in $0.3 \%$ hydrogen peroxide $\left(\mathrm{H}_{2} \mathrm{O}_{2}\right)$ for $15 \mathrm{~min}$. The slides were then immersed in $10 \%$ goat serum to block unspecific background staining and incubated with primary antibodies for $1 \mathrm{~h}$ at room temperature. The primary antibody against mouse TF (polyclonal IgG rabbit antibody; American Diagnostica, Greenwich, CT, USA) was diluted to 1:100, and the primary antibody against human TF (4509 monoclonal mouse antibody; American Diagnostica) to
1:2000. The secondary antibody was biotin conjugated, goat anti-rabbit IgG (Vector Laboratories, Burlingame, CA, USA), and the chromagen was 3,3'-diaminobenzidine (Biocare Medical, Concord, CA, USA). The slides were counterstained with $1 \%$ methyl green. IHC was performed three times for each specimen (the eyes and the cultured cells). Representative images were taken for illustrations.

\section{LPS and $\mathrm{H}_{2} \mathrm{O}_{2}$ Stimulation of ARPE-19 Cells}

ARPE-19 cells grown to $90 \%$ confluence in $25 \mathrm{~cm}^{2}$ flasks were incubated in fresh growth medium without FBS for $24 \mathrm{~h}$, and subsequently with various concentrations of LPS and $\mathrm{H}_{2} \mathrm{O}_{2}$. In all, $5 \mathrm{mg}$ Salmonella typhimurium LPS stock (DIFCO, Livonia, MI, USA) was used at final concentrations of 1,5 , and $10 \mu \mathrm{g} / \mathrm{ml}$ in growth media for $24 \mathrm{~h}$. In order to induce oxidative stress, 100 and $200 \mu \mathrm{M} \mathrm{H}_{2} \mathrm{O}_{2}$-conditioned media was added for $2 \mathrm{~h}$ by dissolving $30 \% \mathrm{H}_{2} \mathrm{O}_{2}$ stock solution (Fisher Scientific, Fair Lawn, NJ, USA) in growth media just before use. Afterwards, the stimulated cells were examined for TF mRNA and protein expression via RT-PCR and IHC, as described above.

\section{Determination of ARPE-19 Cell Viability by Crystal Violet Staining Method}

ARPE-19 cells were seeded in 96-well culture plate in growth medium with FBS. After the cells became adherent, growth medium without FBS was added for $24 \mathrm{~h}$. After LPS and $\mathrm{H}_{2} \mathrm{O}_{2}$ stimulation for the indicated time periods, the cells were washed with phosphate-buffered saline (PBS), stained with crystal violet staining solution $(0.4 \%$ crystal violet, $20 \%$ ethanol) for $20 \mathrm{~min}$, washed three times with PBS, and air-dried for $1 \mathrm{~h}$. The cells were then incubated with $200 \mu \mathrm{l}$ of extraction buffer (10\% methanol, $10 \%$ acetic acid) for $15 \mathrm{~min}$. The dye mixture $(100 \mu \mathrm{l})$ was transferred to a 96-well plate, and viability was determined by measuring the optical density at $590 \mathrm{~nm}$ using an ELISA plate reader (BioTek, Burlington, VT, USA). The cell viability was $>90 \%$ in all experiments.

\section{Statistical Analysis}

Differences in $T F$ mRNA levels among groups were evaluated by one-way analysis of variance (ANOVA) using SPSS version 16.0 (SPSS, Chicago, IL, USA) with the level of significance set at $P<0.05$. The values are presented as means \pm s.d.

\section{RESULTS}

\section{Detection of TF Expression in Human AMD Retina}

IHC result showed diffuse immunoreactivity for TF, which was expressed in the entire neuroretina of both non-AMD (Figure 1a) and AMD (Figure 1b) eyes, in particular in the inner and outer plexiform layers as well as in the nerve fiber layers (see the arrows) as reported previously. ${ }^{14}$ However, immunoreactivity for TF was much stronger in AMD retina when compared with non-AMD retina. Within the same eye, the macula showed higher TF expression than the periphery 

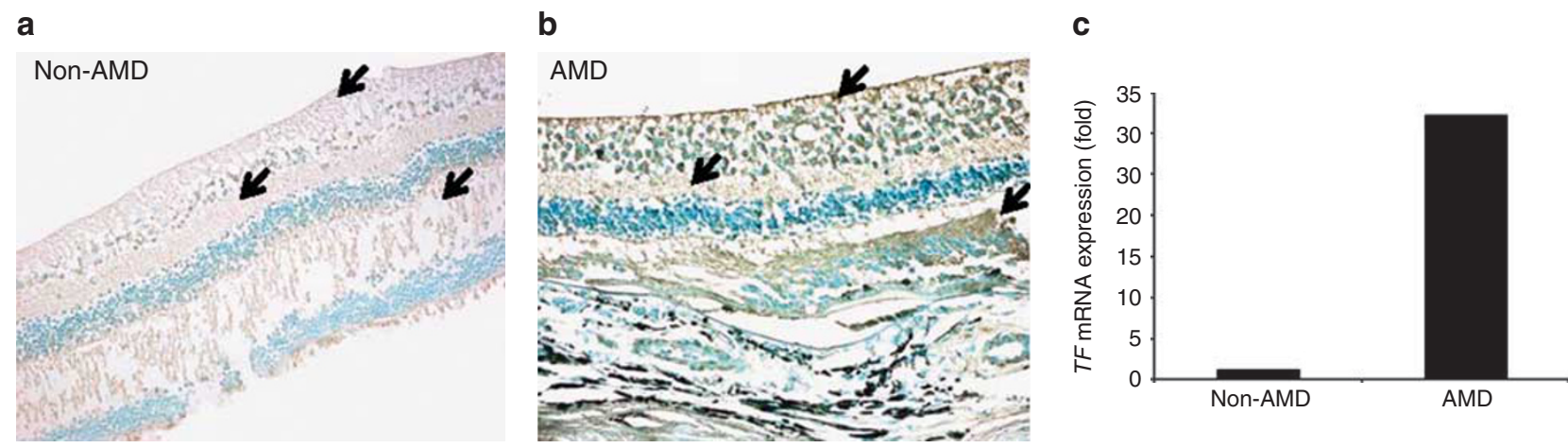

Figure 1 TF expression in human non-AMD and AMD retina. A representative section stained with the avidin-biotin complex method is illustrated (a) in human non-AMD retina and (b) in human AMD retina (advanced wet AMD). In all samples evaluated to date, IHC analysis shows more intense immunoreactivity against TF (brown-blackish staining indicated by arrows) in AMD compared with non-AMD retina. (c) RT-PCR was performed on one AMD retina and one non-AMD retina to quantify relative TF mRNA expression. Microdissected retinal lesions in AMD retina demonstrated a 32-fold increase in TF transcript level when compared with normal maculae from non-AMD retina.

(data not shown). Detectable RT-PCR result was only available for microdissected cells from one non-AMD and one advanced wet AMD retina. The expression of the TF transcript in AMD lesions was 32-fold higher than the expression level in the non-AMD normal retinal area (Figure 1c).

\section{Evaluation of TF Expression in WT and DKO Mouse Eyes}

Knowing that elevated TF expression might be associated with the pathogenesis of AMD, we investigated whether TF expression is also increased in the eyes from the DKO murine model of AMD. Frozen sections of age-matched WT and DKO mouse retina were immunostained for TF expression (Figures 2a and b, respectively). Moderately higher TF expression was observed in all cells of the DKO retina when compared with those in the WT retina; in particular, the plexiform layers and the nerve fiber layer were most heavily endowed with TF expression (see the asterisks).

In order to investigate whether TF is particularly upregulated in the retinal lesion sites and, therefore, potentially contributes to AMD pathogenesis, retinal cells in the lesions were microdissected from frozen sections of age-matched WT and DKO retina for quantitative RT-PCR (Figure 2c). The frozen sections and funduscopic pictures of the second samples of DKO retina demonstrated more lesions than the first DKO samples (data not shown). The RT-PCR results demonstrated a trend toward higher TF mRNA expression in DKO lesions compared with WT normal retina, which was more prominent in the second samples of mice. In the first samples of mice, the expression of TF in young and old DKO mice was 1.93- and 4.69-fold greater, respectively, when compared with the expression level observed in the retina of young WT mice, whereas the second samples of young and old DKO mice demonstrated 9.00- and 20.95-fold increase, respectively, in $T F$ transcript level compared with that in young WT mice from the first samples of experiments. In addition, $T F$ transcript levels increased in an age-related fashion in both WT and DKO mice, indicating a possible link between age and TF expression in the retina.

The whole neuroretinal tissue (a much larger surface area than the microdissected retinal cells in the focal lesions) was removed from age-matched DKO and WT mice for RNA isolation to evaluate generalized $T F$ expression level (Figure 2d). TF mRNA level increased significantly as a function of age in both WT and DKO mice, from 1.00 to $2.35 \pm 0.1$ in WT $(P<0.01)$ and from $1.46 \pm 0.50$ to $3.39 \pm 0.59$ in DKO mice $(P<0.05)$. The older subgroup of DKO mice had a significantly higher mean $T F$ mRNA level than was observed in the older WT mice $(P<0.05)$. TF mRNA was increased slightly in young DKO mice compared with young WT mice, but this difference was not statistically significant.

Last, mice RPE cells were dissected and cultured for IHC and RT-PCR. Compared with age-matched WT RPE cells, RPE cells from both young and old subgroups of DKO mice expressing significantly higher $T F$ transcript levels were observed in both young $(P<0.05)$ and old $(P<0.05)$ subgroups of DKO (Figure 2e; $n=3$ ). Stronger immunoreactivity for TF was observed in DKO RPE cells (Figure $2 \mathrm{~g}$ ) when compared with WT RPE cells (Figure 2f). Similar to the previous results, TF mRNA level in RPE cells showed a positive association with age in both WT and DKO mice, increasing from 1.00 to $6.53 \pm 2.42$ in cells from the WT mice and from $3.39 \pm 2.57$ to $10.47 \pm 1.19$ in cells from the DKO mice. However, this difference was not statistically significant.

\section{LPS and $\mathrm{H}_{2} \mathrm{O}_{2}$-Mediated Induction of TF Expression in ARPE-19 Cells}

ARPE- 19 cells were stimulated with 1,5 , and $10 \mu \mathrm{g} / \mathrm{ml}$ of LPS for $24 \mathrm{~h}$. Moderately increased TF antigen expression was observed in ARPE-19 cells stimulated with $10 \mu \mathrm{g} / \mathrm{ml}$ LPS (Figure 3b) compared with nonstimulated control cells (Figure 3a). TF mRNA expression in the cultured cells demonstrated a dose-dependent increase with LPS treatment (Figure 3c). LPS doses of 1,5 , and $10 \mu \mathrm{g} / \mathrm{ml}$ induced, 
a
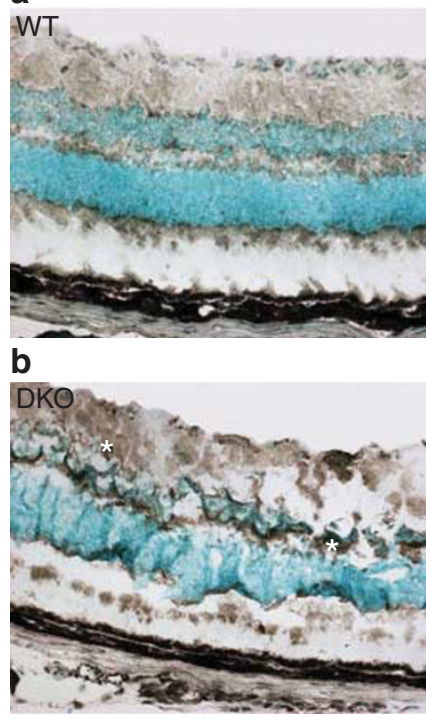

C

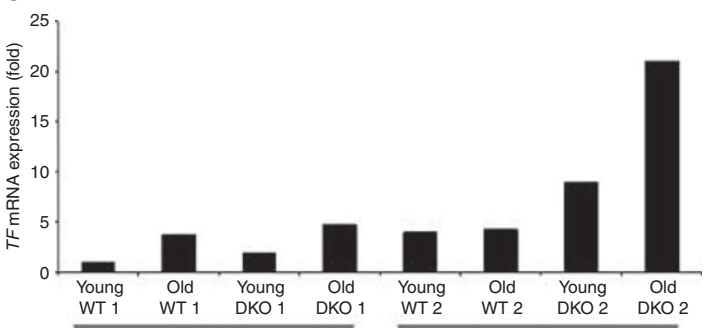

d

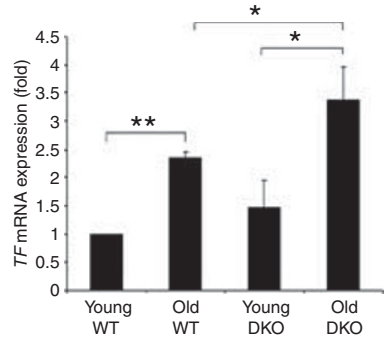

e

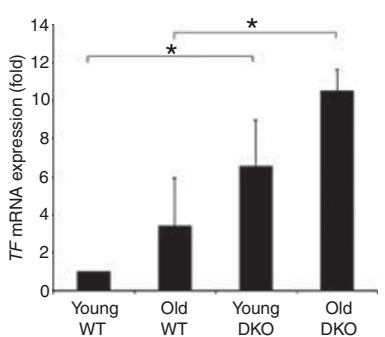

f

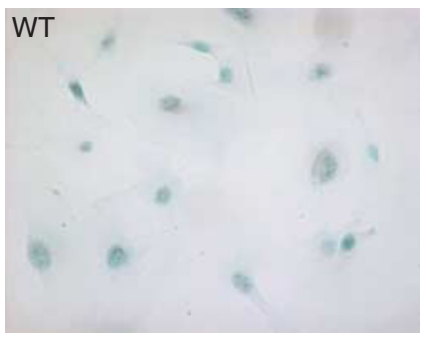

g

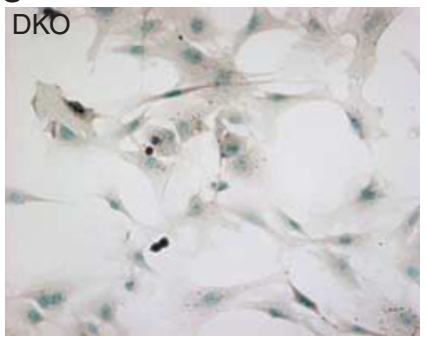

Figure 2 TF expression in WT and DKO mouse eyes. (a, b) IHC demonstrated more intense TF staining (asterisks) in DKO retina compared with WT retina. (c) TF mRNA levels were analyzed and compared with that in young WT. Retinal lesions of DKO mice express higher TF mRNA levels than is observed in the normal retinal area of age-matched WT mice. Two sets of mice were analyzed and the retinal tissue from the second set of DKO mice (with more lesions) exhibited a higher TF expression level than was observed with the first set of animals. (d) TF mRNA expression in neuroretinal tissue increased in DKO mice when compared with age-matched WT mice. TF expression increased with age in WT and DKO mouse retina $\left({ }^{*} P<0.05\right.$; ${ }^{*} P<0.01 ; n=3$ ). (e) TF mRNA expression in RPE cells isolated from the mice also increased with age. DKO RPE cells demonstrated significantly higher TF transcript level compared with age-matched WT cells $\left({ }^{*} P<0.05 ; n=3\right)$. (f, g) TF antigen expression was elevated in cultured RPE cells isolated from WT mice compared with RPE cells isolated from DKO mice.

respectively, $2.01 \pm 1.27-, 2.17 \pm 0.15-$, and $3.45 \pm 0.16$-fold increase in TF transcript level in the cells. The results of the 5 and $10 \mu \mathrm{g} / \mathrm{ml}$ doses of LPS stimulation were found to be statistically significant $(P<0.05$ and $P<0.01$, respectively).

Stimulation with $200 \mu \mathrm{M} \mathrm{H}_{2} \mathrm{O}_{2}$ for $2 \mathrm{~h}$ (Figure 3e) did not induce noticeable TF protein expression compared with the control cells (Figure 3d). TF mRNA expression was increased $1.30 \pm 0.44$ - and $1.52 \pm 0.69$-fold over the control cells (Figure 3f) when stimulated by 100 and $200 \mu \mathrm{M} \mathrm{H} \mathrm{H}_{2} \mathrm{O}_{2}$, respectively. No statistical significance was observed.

Finally, ARPE- 19 cells prestimulated with $10 \mu \mathrm{g} / \mathrm{ml}$ LPS for $24 \mathrm{~h}$ and then induced with previously selected doses of $\mathrm{H}_{2} \mathrm{O}_{2}$ for $2 \mathrm{~h}$ demonstrated increased TF protein expression (Figure $3 \mathrm{~h}$ ) compared with control (Figure 3g). RT-PCR data showed that $10 \mu \mathrm{g} / \mathrm{ml}$ LPS treatment alone induced $3.88 \pm 1.07$-fold significant increase in $T F$ transcript expression (Figure 3i; $P<0.01$ ). Addition of 100 and $200 \mu \mathrm{M} \mathrm{H}_{2} \mathrm{O}_{2}$ induced a further increase in TF mRNA expression levels to $4.51 \pm 0.35$ $(P<0.01)$ and $4.95 \pm 0.60(P<0.01)$, respectively (Figure $3 \mathrm{i})$. These data suggest that enhanced TF expression in human AMD and DKO eyes may be, in part, induced by inflammatory signals (eg, simulated by LPS) and oxidative stress (eg, simulated by $\mathrm{H}_{2} \mathrm{O}_{2}$ ).

\section{DISCUSSION}

Tissue factor is a ubiquitous cell-surface protein that regulates the activation of mammalian coagulation. TF is expressed constitutively only on the surface of cells involved in so-called 'barrier functions'-for example, fibroblasts, smooth muscle cells, pericytes, trophoblasts, and so on-in anatomic locations where the prevention of bleeding is critical (eg, subendothelium, central nervous system, lung, placenta, and, perhaps, in the eye). In the membrane of other cells, such as endothelial cells and circulating monocytes, TF exists in an encrypted form, devoid of procoagulant properties. In response to specific receptor binding to TF of its natural, high-affinity ligand (FVII and/or FVIIa), or to cell stimulation by inflammatory mediators such as IL- $1 \beta$ or TNF- $\alpha$, and/or interaction with proteases released from inflammatory or malignant cells, cellular TF becomes 'deencrypted' and is able to function as an important regulator of signal transduction. Indeed, TF expression has now been linked to oncogene-mediated malignant transformation of cells, atherogenesis, chronic inflammation, and a variety of other pathogenic processes. ${ }^{27,28}$ It was with this knowledge of the rather ubiquitous relationship of TF expression with inflammatory, degenerative, and malignant processes that we sought information regarding TF in AMD.

To our knowledge, this is the first study that demonstrates the association of intraocular TF expression with increasing age, and compares TF expression levels of normal with AMD eyes, as well as WT and DKO eyes. ARPE-19 cells were also used as an in vitro model in order to understand better how the microenvironment of AMD might induce TF expression. Although TF has been implicated in AMD, ${ }^{14}$ in most of the published studies, investigators have examined TF in human 
a

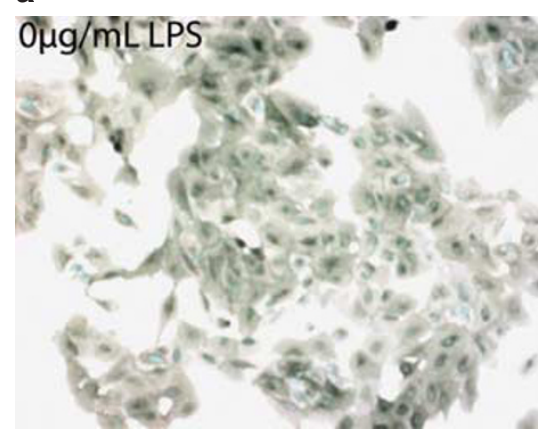

b
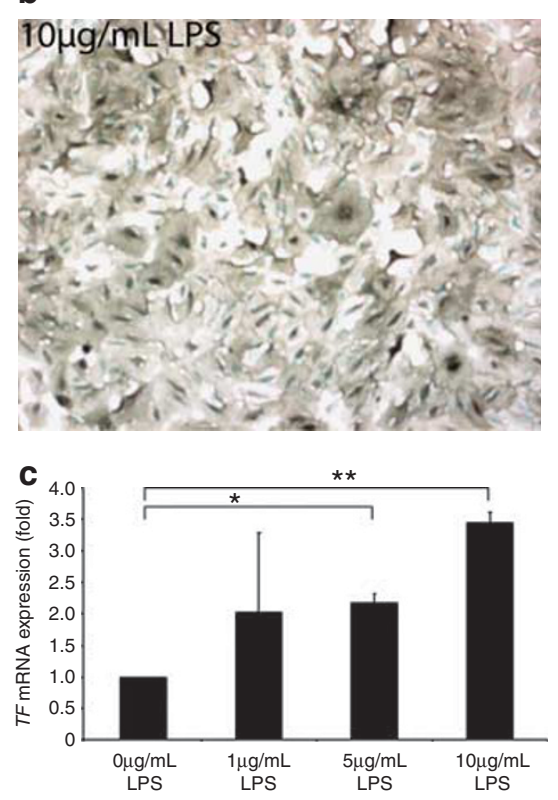

d

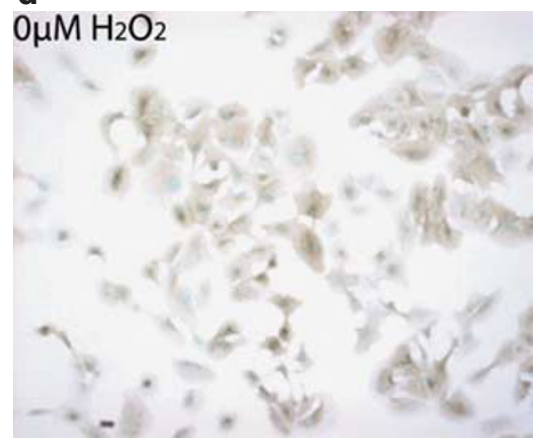

e
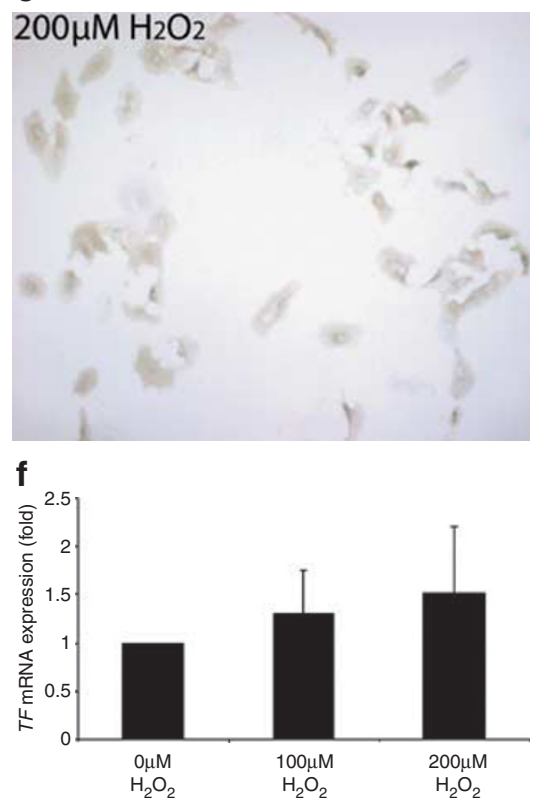

g

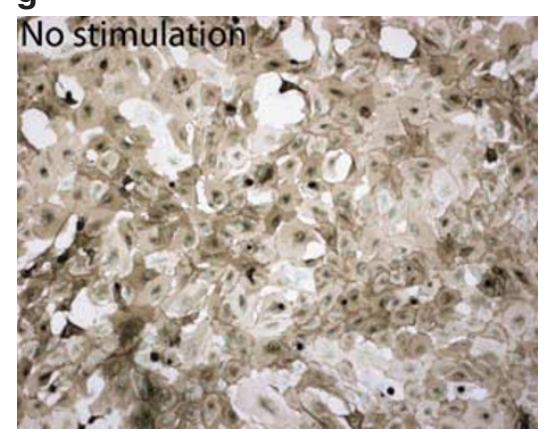

h
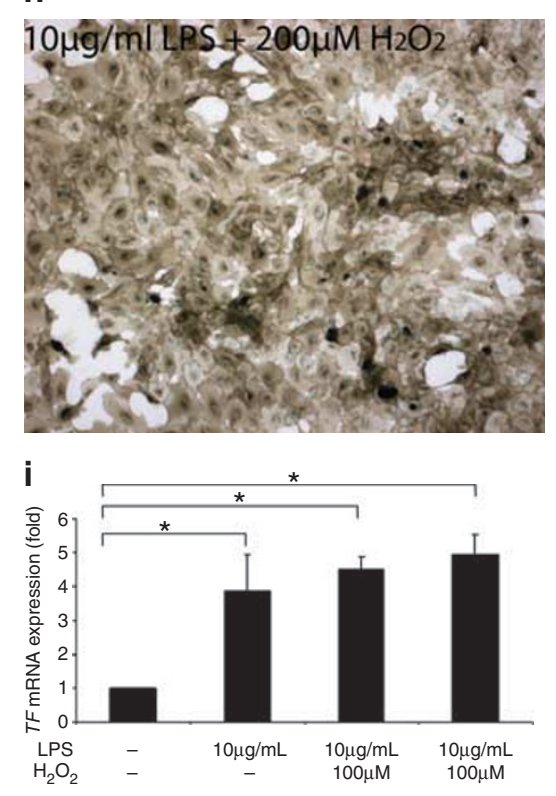

Figure 3 TF expression in ARPE-19 cells. (a, b) ARPE-19 cells stimulated with $10 \mu \mathrm{g} / \mathrm{ml}$ LPS demonstrated increased TF protein expression compared with nonstimulated control cells. (c) Dose-response curve for LPS; increased TF mRNA expression with increasing concentration of LPS $(* P<0.05$; $* * P<0.01$; $n=3$ ). There was no statistically significant difference among 1, 5, and $10 \mu$ g treatments. (d, e) TF protein expression in ARPE-19 cells increased when stimulated with $\mathrm{H}_{2} \mathrm{O}_{2}$ compared with control cells. (f) $T F$ mRNA expression slightly increased upon $\mathrm{H}_{2} \mathrm{O}_{2}$ stimulation ( $n=3$ ). (g, h) Stimulation with $10 \mu \mathrm{g}$ LPS and $200 \mu \mathrm{M} \mathrm{H}_{2} \mathrm{O}_{2}$ induced a moderate increase in TF protein expression compared with the control. The result of the combination of stimulants was similar to that of LPS treatment alone. (i) LPS alone increased TF mRNA expression by 3.88-fold over the baseline level, similar to the previous results. Addition of $\mathrm{H}_{2} \mathrm{O}_{2}$ further enhanced $T F$ transcript level but the difference was not statistically significant $\left({ }^{\star} P<0.01\right.$; $n=3$ ).

$\mathrm{CNV}$ specimens and laser-induced CNV models, which reflect principally the neovascular component of AMD. ${ }^{21-23}$ In addition, it remains unclear whether TF expression induced by laser injury has direct relevance to evaluating the role of TF expression in AMD or simply represents a response to acute injury or wound healing regardless of underlying retinal disease.

Enhanced expression of TF mRNA and protein was observed in human advanced wet AMD retina compared with non-AMD retina (Figures $1 \mathrm{a}-\mathrm{c}$ ). Due to the availability of a limited number of AMD cases, we cannot be certain if our findings are representative of all AMD cases; a question that remains unanswered and requires validation. As an alternative, we examined TF expression in the human RPE cell line, ARPE-19, after creating an environment that mimics that of AMD, which will be discussed later.
In this study, use of the $C \mathrm{cl} 2^{-/-} / \mathrm{C} \times 3 \mathrm{cr} 1^{-/-}$murine model of retinal lesions allowed more definitive investigation of the potential association of TF expression with early and also likely dry AMD. DKO mice spontaneously develop retinal lesions without exogenous stimulation, and thus may provide a model, which may closely represent the likely expression and distribution of TF in AMD, in the absence of additional variables such as physical injury and the wound healing response. Therefore, the DKO model enables us to define the effects of age on retinal TF expression independent of laser photocoagulation-induced injury. WT and DKO mice were divided into young and old groups to explore the potential association of TF expression with increasing age. A similar age-dependent increase in TF expression was observed in WT and DKO neuroretinal tissue (Figures $2 \mathrm{c}-\mathrm{e}$ ), which was statistically significant (Figures $2 \mathrm{~d}$ and e). The apparently higher 
TF transcript levels in young DKO vs young WT mice were not significantly different, whereas the pairwise comparison in old DKO vs old WT mice was significantly different. As young DKO mice start to develop lesions at 4-6 weeks of age and old DKO mice show confluent lesions and large areas of photoreceptor atrophy, ${ }^{24,25}$ it may be reasonable to postulate that TF expression in the neuroretinal tissue does not increase in the early stage of lesion development but becomes significant in later stages.

Moderately higher TF antigen expression was observed in DKO mice (Figure $2 \mathrm{~b}$ ), indicating a potential link between TF and AMD. Furthermore, TF mRNA level closely corresponded to the number and severity of retinal lesions in DKO mice; the first samples of young and old DKO mice presented with a small focal lesion and retinal degeneration, respectively, whereas the second samples of young and old DKO mice had three focal lesions with RPE vacuolization, photoreceptor degeneration and atrophy, respectively. AMD is a focal disease in which alterations in gene expression in lesion tissue appears to be highly relevant for determining the pathogenic mechanisms of the disease. Therefore, upregulated TF expression in the retinal lesions suggests that TF may have a role in AMD pathogenesis.

Cultured RPE cells dissected from age-matched WT and DKO eyes also demonstrated an age-related increase in TF transcript level. Both young and old DKO RPE cells showed statistically significant increases in $T F$ transcript levels when compared with age-matched WT RPE cells. RPE cells have been reported to express variable levels of TF in human CNV specimens. ${ }^{21}$ Our data confirm that RPE cells are capable of expressing TF, and extend previous observations by demonstrating that TF may be involved in both dry and wet AMD pathology.

Given that inflammatory and oxidative stress, the key contributors to aging and AMD, are capable of inducing TF expression, ARPE-19 cells were stimulated with LPS (inflammation) and $\mathrm{H}_{2} \mathrm{O}_{2}$ (oxidative stress) to simulate a microenvironment consistent with that of AMD and investigate these effect on TF expression. LPS has been used to induce TF expression in monocytes, platelets, and human umbilical vein endothelial cells. ${ }^{29-32}$ In ARPE-19 cells, LPS consistently enhanced TF protein and transcript expression, confirming that a proinflammatory environment can induce TF expression in these cells from the eye as well.

Compared with LPS treatment, $\mathrm{H}_{2} \mathrm{O}_{2}$ stimulation induced a slight increase in $T F$ mRNA and protein level (Figures $3 \mathrm{~d}-$ f). Both human and murine TF genes contain one NF- $\kappa \mathrm{B}$ and two AP-1-binding sites, which are required for optimal gene transcription, ${ }^{33-36}$ and $\mathrm{H}_{2} \mathrm{O}_{2}$ is known to induce the translocation of NF- $\kappa \mathrm{B}$ and initiate AP-1 binding. However, Penn et $a l,{ }^{37}$ using smooth muscle cell culture, also observed that $\mathrm{H}_{2} \mathrm{O}_{2}$ did not significantly increase TF mRNA or cell surface $\mathrm{TF}$ antigen levels, but rather enhanced the activity of preexisting, latent TF. It is noteworthy that apoptosis is known as an important contributor to blood or plaque thrombogenicity, and colocalization of cellular and extracellular TF expression with apoptotic death was found in the lipid core of atherosclerotic plaques. ${ }^{38}$ In AMD, apoptotic processes in RPE and photoreceptors induced by oxidative stress may contribute to enhanced TF expression. Thus, different cell types and sources of oxidative stress, as well as duration and severity of the stress, seem to have a role in regulating TF expression and activity.

Although we have limited studies directly linking TF to AMD, several pathological roles of TF in AMD can be proposed based on previous studies of TF expression in other analogous human diseases. Increased TF expression is a hallmark of many inflammatory conditions, such as sepsis, atherosclerosis, and the antiphospholipid syndrome. Redecha et $a l^{39}$ showed that anaphylatoxin C5a, which is also observed in drusen, induced TF expression on the surface of neutrophils in antiphospholipid-treated mice. Enhancement of ROS production by TF has been described in human macrophages. ${ }^{40}$ Furthermore, inflammation-active human CNV showed much more intense TF reactivity than inflammationinactive CNV. ${ }^{21}$ Thus, increased TF expression in AMD may induce RPE and photoreceptor damage by upregulating both inflammation and ROS production.

Previous studies on the pathobiological role of TF in inflammation and malignancy suggest that enhanced $T F$ gene expression, perhaps with activation of localized blood coagulation, may contribute to AMD development. However, whether activation of TF signaling is also relevant to AMD has not yet been determined. TF was shown to be selectively phosphorylated in association with pathological neovascular but not normal vessels in diabetic retinopathy. ${ }^{6}$ In order to identify whether TF is an essential participating factor in the pathogenesis of AMD, it will be important to evaluate the level of TF phosphorylation in AMD and DKO retina, in addition to TF expression. In the present studies, we have demonstrated that TF expression increases in human AMD maculae and DKO mouse eyes compared with non-AMD maculae and WT eyes, and that upregulated TF expression may be associated with AMD. Our data also suggest that inflammatory and oxidative stress may lead to enhanced TF expression in aging eyes as well as AMD eyes. A more complete understanding of the role of TF expression and function in AMD may provide a further rationale for potentially targeting TF in the therapy of AMD.

\section{ACKNOWLEDGEMENT}

This study was supported by the Intramural Research Program of the National Eye Institute, National Institutes of Health.

\section{DISCLOSURE/CONFLICT OF INTEREST}

Dr Rickles declares a proprietary interest in the development of TF-targeting therapies by Pharmacyclics and Genmab. The other authors declare no conflict of interest.

1. Coleman HR, Chan CC, Ferris III FL, et al. Age-related macular degeneration. Lancet 2008;372:1835-1845.

2. Ding X, Patel M, Chan CC. Molecular pathology of age-related macular degeneration. Prog Retin Eye Res 2009;28:1-18. 
3. Drake TA, Morrissey JH, Edgington TS. Selective cellular expression of tissue factor in human tissues. Implications for disorders of hemostasis and thrombosis. Am J Pathol 1989;134:1087-1097.

4. Chu AJ. Tissue factor mediates inflammation. Arch Biochem Biophys 2005;440:123-132.

5. Chu AJ. Role of tissue factor in thrombosis. Coagulation-inflammationthrombosis circuit. Front Biosci 2006;11:256-271.

6. Belting M, Dorrell MI, Sandgren $\mathrm{S}$, et al. Regulation of angiogenesis by tissue factor cytoplasmic domain signaling. Nat Med 2004;10:502-509.

7. Chen J, Bierhaus A, Schiekofer S, et al. Tissue factor-a receptor involved in the control of cellular properties, including angiogenesis. Thromb Haemost 2001;86:334-345.

8. Belting $M$, Ahamed J, Ruf W. Signaling of the tissue factor coagulation pathway in angiogenesis and cancer. Arterioscler Thromb Vasc Biol 2005;25:1545-1550.

9. de Jong PT. Age-related macular degeneration. $\mathrm{N}$ Engl J Med 2006;355:1474-1485.

10. Anderson DH, Talaga KC, Rivest AJ, et al. Characterization of beta amyloid assemblies in drusen: the deposits associated with aging and age-related macular degeneration. Exp Eye Res 2004;78:243-256.

11. Crabb JW, Miyagi M, Gu X, et al. Drusen proteome analysis: an approach to the etiology of age-related macular degeneration. Proc Natl Acad Sci USA 2002;99:14682-14687.

12. Johnson LV, Leitner WP, Staples MK, et al. Complement activation and inflammatory processes in Drusen formation and age related macular degeneration. Exp Eye Res 2001;73:887-896.

13. Mullins RF, Russell SR, Anderson $\mathrm{DH}$, et al. Drusen associated with aging and age-related macular degeneration contain proteins common to extracellular deposits associated with atherosclerosis, elastosis, amyloidosis, and dense deposit disease. FASEB J 2000;14:835-846.

14. Cho Y, Rickles FR, Parver LM, et al. The potential pathophysiological role of tissue factor in age-related macular degeneration. Expert Rev Ophthalmol 2010;5:27-34.

15. Chu AJ. Tissue factor upregulation drives a thrombosis-inflammation circuit in relation to cardiovascular complications. Cell Biochem Funct 2006;24:173-192.

16. Yang XM, Wang YS, Zhang J, et al. Role of PI3K/Akt and MEK/ERK in mediating hypoxia-induced expression of HIF-1alpha and VEGF in laser-induced rat choroidal neovascularization. Invest Ophthalmol Vis Sci 2009;50:1873-1879.

17. Abe K, Shoji M, Chen J, et al. Regulation of vascular endothelial growth factor production and angiogenesis by the cytoplasmic tail of tissue factor. Proc Natl Acad Sci USA 1999:96:8663-8668.

18. Zhang $Y$, Deng $Y$, Luther $T$, et al. Tissue factor controls the balance of angiogenic and antiangiogenic properties of tumor cells in mice. J Clin Invest 1994;94:1320-1327.

19. Chiarugi V, Ruggiero M, Magnelli L. Molecular polarity in endothelial cells and tumor-induced angiogenesis. Oncol Res 2000;12:1-4.

20. Takano S, Tsuboi $\mathrm{K}$, Tomono $\mathrm{Y}$, et al. Tissue factor, osteopontin alphavbeta3 integrin expression in microvasculature of gliomas associated with vascular endothelial growth factor expression. $\mathrm{Br}$ Cancer 2000;82:1967-1973.

21. Grossniklaus HE, Ling JX, Wallace TM, et al. Macrophage and retinal pigment epithelium expression of angiogenic cytokines in choroidal neovascularization. Mol Vis 2002;8:119-126.

22. Bora PS, $\mathrm{Hu} \mathrm{Z}$, Tezel $\mathrm{TH}$, et al. Immunotherapy for choroidal neovascularization in a laser-induced mouse model simulating exudative (wet) macular degeneration. Proc Natl Acad Sci USA 2003; 100:2679-2684.

23. Tezel $\mathrm{TH}$, Bodek $\mathrm{E}$, Sonmez $\mathrm{K}$, et al. Targeting tissue factor for immunotherapy of choroidal neovascularization by intravitreal delivery of factor VII-Fc chimeric antibody. Ocul Immunol Inflamm 2007; 15:3-10.

24. Tuo J, Bojanowski $C M$, Zhou $M$, et al. Murine $\mathrm{ccl} 2 / \mathrm{cx} 3 \mathrm{cr} 1$ deficiency results in retinal lesions mimicking human age-related macular degeneration. Invest Ophthalmol Vis Sci 2007;48:3827-3836.

25. Chan CC, Ross RJ, Shen D, et al. Ccl2/Cx3cr1-deficient mice: an animal model for age-related macular degeneration. Ophthalmic Res 2008;40:124-128.

26. Verma V, Sauer T, Chan CC, et al. Constancy of ERp29 expression in cultured retinal pigment epithelial cells in the $\mathrm{Ccl} 2 / \mathrm{Cx} 3 \mathrm{cr} 1$ deficient mouse model of age-related macular degeneration. Curr Eye Res 2008;33:701-707.

27. Dvorak HF, Rickles FR. Malignancy and hemostasis. In: Colman RW, Hirsh J, Marder VJ, et al (eds) Hemostasis and Thrombosis: Basic Principles and Clinical Practice, 5th edn Lippincott-Raven: Philadelphia, PA, 2006, pp 851-873.

28. Rickles FR. Mechanisms of cancer-induced thrombosis in cancer. Pathophysiol Haemost Thromb 2006;35:103-110.

29. Norris LA, Weldon S, Nugent $A$, et al. LPS induced tissue factor expression in the THP-1 monocyte cell line is attenuated by conjugated linoleic acid. Thromb Res 2006;117:475-480.

30. Meszaros K, Aberle S, Dedrick R, et al. Monocyte tissue factor induction by lipopolysaccharide (LPS): dependence on LPS-binding protein and CD14, and inhibition by a recombinant fragment of bactericidal/ permeability-increasing protein. Blood 1994;83:2516-2525.

31. Stephens AC, Ranlall NF, Rivers RP. Suppression of HUVEC tissue factor synthesis by antisense oligodeoxynucleotide. Thromb Res 2008;122:99-107.

32. Panes $O$, Matus $V$, Saez CG, et al. Human platelets synthesize and express functional tissue factor. Blood 2007;109:5242-5250.

33. Brisseau GF, Dackiw AP, Cheung PY, et al. Posttranscriptional regulation of macrophage tissue factor expression by antioxidants. Blood 1995;85:1025-1035.

34. Mackman N. Regulation of tissue factor gene expression in human monocytic and endothelial cells. Haemostasis 1996;26 (Suppl 1):17-19.

35. Oeth P, Parry GC, Mackman N. Regulation of the tissue factor gene in human monocytic cells. Role of AP-1, NF-kappa B/Rel, and Sp1 proteins in uninduced and lipopolysaccharide-induced expression. Arterioscler Thromb Vasc Biol 1997;17:365-374

36. Moll T, Czyz M, Holzmuller $H$, et al. Regulation of the tissue factor promoter in endothelial cells. Binding of NF kappa B-, AP-1-, and Sp1like transcription factors. J Biol Chem 1995;270:3849-3857.

37. Penn MS, Patel CV, Cui MZ, et al. LDL increases inactive tissue factor on vascular smooth muscle cell surfaces: hydrogen peroxide activates latent cell surface tissue factor. Circulation 1999;99:1753-1759.

38. Mallat Z, Tedgui A. Current perspective on the role of apoptosis in atherothrombotic disease. Circ Res 2001;88:998-1003.

39. Redecha $\mathrm{P}$, Tilley $\mathrm{R}$, Tencati $\mathrm{M}$, et al. Tissue factor: a link between $\mathrm{C} 5 \mathrm{a}$ and neutrophil activation in antiphospholipid antibody induced fetal injury. Blood 2007;110:2423-2431.

40. Cunningham MA, Romas $P$, Hutchinson $P$, et al. Tissue factor and factor VIla receptor/ligand interactions induce proinflammatory effects in macrophages. Blood 1999;94:3413-3420. 